\title{
Moderating supplementary data
}

\author{
Nature Neuroscience announces changes to its supplementary information guidelines and expands the space \\ allowed for methods.
}

S upplementary information (SI) has been a focus of much discussion in the scientific community in the last few years. Although some journals have done away with SI completely, we continue to recognize the value of supplementary data. SI provides a repository to present supporting data such as movies, audio files or large datasets that cannot be accommodated in the main article. Furthermore, it can provide aficionados in the field with the same supporting data that the referees were provided, such as full-length gels or blots, controls for antibodies, detailed information on constructs or sample preparation, or a comprehensive description of mathematical modeling. However, unrestrained SI can be problematic for readers, authors, referees and editors. Authors complain that SI has evolved into a convenient instrument for referees to ask for more data that is only marginally related to the main story. For referees, the growth of SI has also added considerably to their workload and, in some cases, has made it difficult to find all of the information necessary to fully evaluate a manuscript. Although the general reader may not ever glance at the SI, for specialists, the proliferation of SI has made the reading of the final paper confusing and unwieldy, and authors have been known to deposit all kinds of ancillary materials into the SI, including experiments that don't fit with the main paper, data that don't directly speak to their main hypothesis or simply text that was cut to conform to the journal length limits.

We recognize that there are no easy solutions to this problem. There are good uses for SI, but it seems clear that SI will need to be moderated by all of the participants in the process, authors, referees and editors, to present a coherent and self-contained paper. We recognize that, particularly for specialist readers, integrating the SI meaningfully into the published paper can be invaluable. However, before we can do this, we feel that editors (sometimes in concert with referees) and authors need to have a more active dialog in critically evaluating the scope and quantity of SI. This month we are rolling out new guidelines for supplementary material that we hope will represent a first step in the right direction and that will help us shape future guidelines.

One first step is to try to limit the amount of SI that authors are allowed to present. For the most part, SI should be limited to data that is directly relevant to the main conclusions of the paper. Certain types of data, such as movies, audio files, large datasets or other data types that cannot be included in the print version of the paper will, out of necessity, have to be included as supplementary material, and no specific restrictions will currently apply to these data types. However, the majority of supplementary material in our articles consists of figures. Although the numbers of such supplementary figures for our papers vary widely, the range for most articles is about 6-12 figures. We therefore feel that a reasonable cap is ten supplementary figures (for all article types); exceptions will only be made in rare cases with editor discretion. We feel that imposing this limit will make it easier for the authors to craft a better paper and will make it more manageable for the referees and editors to evaluate these data.

As a part of these limits, we will no longer allow authors to include text that is simply culled from the main article as 'supplementary methods', 'supplementary discussion' or 'supplementary references'. Methods sections should be integrated into a single, 'online methods' section for both Brief Communications and Articles. Following acceptance, methods will generally be capped at 1,500 words for Brief Communications; for Articles, this section is normally about 2,000 words. In an effort to allow authors to convey all of the essential details, we will also allow authors ten additional references in the methods section.

A second problem that is inherent in the current system of SI is the existence of two separate sets of data that referees and editors must somehow evaluate as a cohesive whole, making it more difficult to determine which data are most critical to the authors' main story. To make this process easier for all parties, when first submitting a paper, we urge all authors to initially provide, as far as possible, one seamless paper, incorporating all of the essential information (including controls and methods) in the main paper. Authors can either highlight or otherwise label the figures they think would be better suited to SI as "Supplementary information $\mathrm{xx}$ " and present it in the paper. We recognize that this will make the main paper longer, but this will hopefully allow authors to craft a better paper. It will also make it easier for referees and editors to evaluate the entire scope of the paper and may avoid instances in which important information is buried in the mass of supplementary data. Once the paper is accepted, we will ask authors to separate out the SI material from the main text before publication. We hope that such a process will make it easier for the editors to conduct a more informed dialog with the authors about what data is truly supplemental.

We appreciate that there is no easy answer to tackling the proliferation of SI and that these new policies may present only a first step. We also recognize that there will be a period of transition in implementing these guidelines and, as always, we will not hold up consideration of a paper because of format. As with all of our initiatives, we welcome your feedback and hope that, with your support, we can work toward better refining the paper to ensure that all the material can be easily organized and evaluated. 